\title{
Caudate Nucleus in Retrieval of Trace Eyeblink Conditioning after Consolidation
}

\author{
Luke C. Flores and John F. Disterhoft \\ Department of Physiology, Feinberg School of Medicine, Northwestern University, Chicago, Illinois 60611
}

Trace eyeblink conditioning (EBC) is an associative learning task in which a stimulus-free trace period separates the presentation of a behaviorally neutral conditioned stimulus (CS; whisker stimulation) from a behaviorally salient unconditioned stimulus (US; air puff to the eye). Repeated pairings of the CS with the US results in the emergence of the conditioned response (CR; eyeblink after CS presentation and before US presentation). The goal of these experiments was to determine whether the caudate nucleus (CN) plays a role in retrieval of previously acquired trace $\mathrm{EBC}$ after memory consolidation. Lesions of the $\mathrm{CN}$ were made 1 month after initial trace $\mathrm{EBC}$. $\mathrm{CN}$-lesioned rabbits performed fewer adaptive CRs and more short-latency non-adaptive responses than sham-lesioned controls. They were not able to improve their CR performance after consolidation as were controls. Single-unit recordings taken from separate cohorts of rabbits demonstrated that neurons in the $\mathrm{CN}$ were still responsive to the CS and US 1 month after initial trace $\mathrm{EBC}$, particularly in the medial and ventral CN on trials when a CR occurred. The proportion of rate-increasing neurons was higher in trace-conditioned than in pseudoconditioned rabbits. Neurons in regions destroyed in the behavioral experiment demonstrated prolonged firing during the trace period, which might underlie the results from the behavioral experiment. These data demonstrate that the $\mathrm{CN}$ plays an important role in retrieval of a previously learned associative task after memory consolidation has occurred.

\section{Introduction}

Eyeblink conditioning (EBC) is an associative learning task in which a behaviorally neutral conditioned stimulus (CS; whisker stimulation) is repeatedly paired with a behaviorally salient unconditioned stimulus (US; air puff directed toward the eye) resulting in the development of a conditioned response (CR; eyeblink after CS presentation but before US presentation). In delay EBC, the CS precedes, overlaps, and coterminates with the US. The cerebellum and brainstem nuclei are required for delay EBC (Clark et al., 1984; Mauk and Thompson, 1987). In trace EBC, a stimulus-free period separates the offset of the CS from the onset of the US. Acquisition of this form of EBC requires forebrain structures, such as the hippocampus (Solomon et al., 1986; Moyer et al., 1990), caudal anterior cingulate cortex (Weible et al., 2000), and primary somatosensory cortex (SI) barrel cortex (Galvez et al., 2007), when whisker stimulation is used as a CS.

Although lesions of the hippocampus made $1 \mathrm{~d}$ after learning criterion impaired trace $\mathrm{EBC}$, lesions made 1 month after had no effect (Kim et al., 1995). In accordance with that finding, CA1 pyramidal neurons in the hippocampus undergo learningrelated changes in neuronal firing during acquisition of trace $\mathrm{EBC}$

Received May 14, 2012; revised Dec. 7, 2012; accepted Dec. 11, 2012.

Author contributions: L.C.F. and J.F.D. designed research; L.C.F. performed research; L.C.F. and J.F.D. analyzed data; L.C.F. and J.F.D. wrote the paper.

This work was supported by National Institutes of Health Grants R01 MH047340, R01 NS059879, and F31 MH081508. We thank Rebekah Ward and Craig Weiss for their generous input to this project.

Correspondence should be addressed to John F. Disterhoft, Department of Physiology, Feinberg School of Medicine, Northwestern University, Chicago, IL 60611. E-mail: jdisterhoft@northwestern.edu.

DOI:10.1523/JNEUROSCI.2326-12.2013

Copyright $\odot 2013$ the authors $\quad 0270-6474 / 13 / 332828-09 \$ 15.00 / 0$
(McEchron and Disterhoft, 1997), and excitability changes can be measured in slice preparations of these neurons after trace EBC but only for 7-14 d (Moyer et al., 1996). These lesion and recording studies suggest that, although the hippocampus is essential for acquisition of trace $\mathrm{EBC}$, it is only a temporary storage buffer and is not required for storage or retrieval of the long-term memory. Lesions of the SI barrel cortex made immediately after trace EBC impaired retention initially, but lesioned rabbits were able to recover to sham-lesioned levels after a few days of training (Galvez et al., 2007), suggesting that the SI barrel cortex plays a limited role in either long-term memory storage or retrieval but can be compensated for by other brain regions. In contrast to these findings from forebrain lesions, muscimol inactivation of the cerebellar interpositus nucleus impaired performance of trace $\mathrm{EBC}$ responses both immediately after and 1 month after initial learning (Pakaprot et al., 2009). Interestingly, evidence for a contribution of extracerebellar regions to longterm memory was observed in rabbits given one session of remote retention training.

The caudate nucleus $(\mathrm{CN})$ is a major input structure for cortical and thalamic input into the basal ganglia network. The basal ganglia are known to be important for learning and memory, especially "stimulus-response" associations (Packard and Knowlton, 2002). In trace EBC, pretraining lesions of the CN prevented acquisition and neurons in the $\mathrm{CN}$ undergo learningrelated changes in firing rate during acquisition (Flores and Disterhoft, 2009). Although the $\mathrm{CN}$ is necessary for acquisition of trace $\mathrm{EBC}$, it may not be critically involved with long-term storage or retrieval of trace $\mathrm{EBC}$. The first experiment was designed to determine whether the $\mathrm{CN}$ is involved in retrieval by performing bilateral electrolytic lesions of the CN 1 month after initial trace 
A

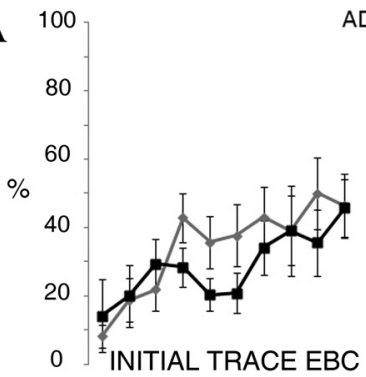

ADAPTIVE CRs

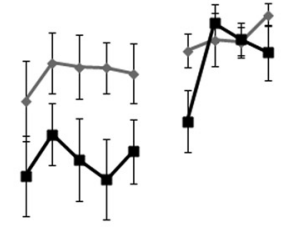

TRACE EBC RETRIEVAL
DELAY EBC $\rightarrow$ Control $\mathrm{N}=7$

$\rightarrow$-Lesion $\mathrm{N}=7$

B
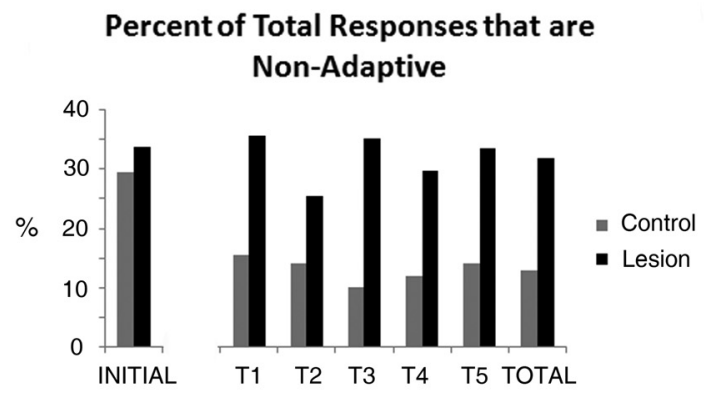

C
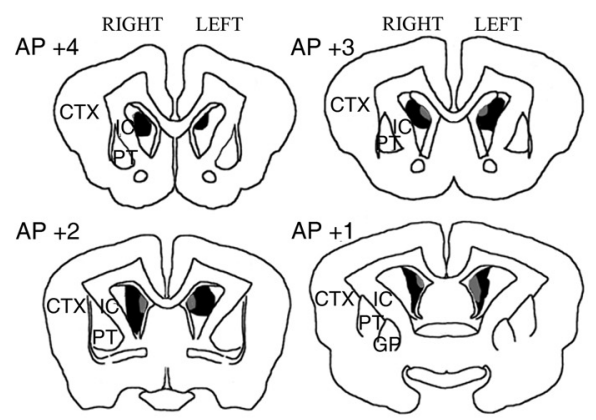

D

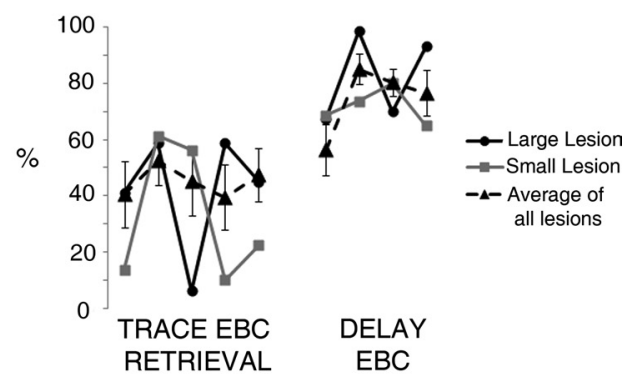

Figure 1. Learning curves of $\mathrm{CN}$-lesioned and sham-lesioned rabbits. $A$, Adaptive CRs performed by $C N$-lesioned and sham-lesioned rabbits during initial trace $E B C$, trace EBC retrieval, and delay EBC. CN-lesioned rabbits performed significantly fewer adaptive CRs during retrieval of trace EBC but not during initial trace EBC or delay EBC. $B$, Percentage of total responses that was non-adaptive. On the final day of initial training, the percentage of non-adaptive responses performed by both control and lesioned rabbits was $>30 \%$. During retrieval sessions $30 \mathrm{~d}$ later, control rabbits performed $<15 \%$ non-adaptive responses, whereas the lesioned rabbits remained $>25 \%$ for all $5 \mathrm{~d}$ of retrieval sessions (T1-T5). The total percentage of non-adaptive responses during retrieval sessions is shown labeled TOTAL. C, Largest (black) and smallest (gray) lesions of the CN. The smallest lesion was focused on the medial aspects of the CN. The largest lesion included medial and ventral regions of the $C N$, leaving the dorsolateral $C N$ essentially intact. $D$, Adaptive $C R s$ performed by the rabbit with the largest lesion (black) and smallest lesion (gray) during retrieval of trace $E B C$ and delay EBC. Both rabbits were impaired during trace EBC retrieval but not delay EBC. CTX, Cortex; IC, internal capsule; GP, globus pallidus; PT, putamen.

EBC, when memory consolidation had presumably occurred. The second experiment was designed to determine the neuronal activity in the $\mathrm{CN}$ during retrieval sessions of trace $\mathrm{EBC} 1$ month after initial training.

\section{Materials and Methods}

Subjects

A total of 14 female New Zealand albino rabbits (3-6 months; average weight, $3.4 \mathrm{~kg}$; ranging from 3.12 to $3.78 \mathrm{~kg}$ ) were used in the lesion experiment. A total of nine female New Zealand albino rabbits (3-6 months; average weight, $2.63 \mathrm{~kg}$; ranging from 2.26 to $3.08 \mathrm{~kg}$ ) were used in the recording experiment. These rabbits were individually housed, provided food and water ad libitum, and maintained on a $12 \mathrm{~h} \mathrm{light/dark}$ cycle. All behavioral tasks were performed during the light cycle. All surgical and behavioral techniques were performed in accordance with Northwestern University Animal Care and Use protocols.

\section{Recording electrode design and construction}

Electrodes were constructed before implantation in the nine rabbits for the recording study. Each tetrode consisted of four Formvar-coated nichrome wires ( $25 \mu \mathrm{m}$ diameter bare, $38 \mu \mathrm{m}$ diameter coated) bound together by spinning the wires together and melting the coating together with a heat gun. The free end of each wire was soldered into a gold-plated Amphenol pin. The tetrodes of each array were passed through a length of 26 gauge hypodermic tubing and glued together. Each electrode array was composed of six tetrodes glued together. Two peripherally mounted drive screws allowed the entire array to be adjusted vertically after implantation.

\section{Surgical procedures}

Head-bolt surgery. All 23 rabbits underwent an initial surgery to affix a head bolt to the skull, which is required for trace EBC. Rabbits were anesthetized with a mixture of ketamine $(60 \mathrm{mg} / \mathrm{kg})$ and xylazine $(10$ $\mathrm{mg} / \mathrm{kg}$ ). Artificial tears was applied to the eyes to keep them moist. The skull was secured in a stereotaxic apparatus with lambda $1.5 \mathrm{~mm}$ below bregma. Under sterile conditions, the skull was exposed, and burr holes were drilled to give access to lesion sites and to implant anchor screws. Dental acrylic was used to build and secure a lightweight headrestraining device containing four nylon bolts to the skull. KwikSeal was used to cover exposed skull over the CN. Rabbits received Buprenex ( 0.3 $\mathrm{mg} / \mathrm{kg})$ immediately after surgery and Metacam $(0.2 \mathrm{mg} / \mathrm{kg})$ immediately after and $24 \mathrm{~h}$ after surgery.

Posttraining lesion surgery. Thirty days after the final day of initial trace $\mathrm{EBC}$, the 14 rabbits in the lesion study were again anesthetized with a mixture of ketamine $(60 \mathrm{mg} / \mathrm{kg})$ and xylazine $(10 \mathrm{mg} / \mathrm{kg})$. Artificial tears was applied to the eyes to keep them moist. The skull was secured in a stereotaxic apparatus with lambda $1.5 \mathrm{~mm}$ below bregma. The skull was exposed by removing the KwikSeal. Holes were drilled at the following sites relative to bregma [anteroposterior (AP), $+3.5 \mathrm{~mm}$ and mediolateral (ML), $\pm 2 \mathrm{~mm}$; AP,$+2 \mathrm{~mm}$ and $\mathrm{ML}, \pm 2.2 \mathrm{~mm} ; \mathrm{AP},+1 \mathrm{~mm}$ and $\mathrm{ML}$, $\pm 2.8 \mathrm{~mm}$ ] for lesion electrode implantation. A sharp lesion electrode was lowered into the $\mathrm{CN}$ (dorsoventral, $-5 \mathrm{~mm}$ measured from dura), and a total of six electrolytic lesions were then made by passing $2 \mathrm{~mA}$ of direct current for $20 \mathrm{~s}$ at each site. In sham-lesioned rabbits, the sharp electrode was lowered into the $\mathrm{CN}$, but no current was passed. Rabbits received Buprenex $(0.3 \mathrm{mg} / \mathrm{kg})$ immediately before surgery and Metacam $(0.2$ $\mathrm{mg} / \mathrm{kg}$ ) immediately after and $24 \mathrm{~h}$ after surgery. Rabbits were given $7 \mathrm{~d}$ to recover.

Posttraining electrode implantation surgery. Thirty days after the final day of initial trace EBC, the nine rabbits of the recording study were again anesthetized with a mixture of ketamine $(60 \mathrm{mg} / \mathrm{kg})$ and xylazine $(10$ $\mathrm{mg} / \mathrm{kg}$ ). Artificial tears was applied to the eyes to keep them moist. The skull was secured in a stereotaxic apparatus with lambda $1.5 \mathrm{~m}$ below bregma. Under sterile conditions, KwikSeal was removed to expose electrode implantation sites. Holes were drilled at the following sites relative to bregma (AP, $+3.5 \mathrm{~mm}$ and $\mathrm{ML}, \pm 2 \mathrm{~mm}$; AP, $+2 \mathrm{~mm}$ and $\mathrm{ML}, \pm 2.2$ $\mathrm{mm}$ ) for recording electrode implantation. The tetrode was lowered until appropriate neuronal activity corresponding to the $\mathrm{CN}$ was seen (typi- 
cally between 4.2 and $5 \mathrm{~mm}$ from dura). Dental acrylic was then used to secure the electrode in place. Rabbits received Buprenex $(0.3 \mathrm{mg} / \mathrm{kg})$ immediately before surgery and Metacam $(0.2$ $\mathrm{mg} / \mathrm{kg}$ ) immediately after and $24 \mathrm{~h}$ after surgery for pain management. Rabbits were given $7 \mathrm{~d}$ to recover from surgery.

\section{Training paradigms}

Trace EBC. All training was performed blind to the rabbits' surgical histories. All 14 rabbits in the lesion study underwent initial trace EBC. Six rabbits in the recording study underwent initial trace EBC. These rabbits received $1 \mathrm{~d}$ of habituation to the testing chamber and behavioral apparatus. Rabbits were placed in cloth bags and secured in a Plexiglas restrainer inside a sound attenuating chamber. The right eye was held open with stainless steel dress hooks, and an infrared sensor/airpuff delivery device was attached to the headrestraining device and aimed $\sim 1 \mathrm{~cm}$ from the right eye. Four or five "B-row" whiskers on the right side of the face were attached to a vibrotactile transducer (T220-A4-303; Piezo Systems) using a thin piece of paper and masking tape. The day after habituation, trace EBC began with the rabbits set up precisely as described during habituation. A computer controlled the delivery of the CS (60 Hz vibration of the whiskers) and the US (3 psi air puff) as well as recorded changes in voltage from the infrared sensor. Each daily session consisted of 80 trials; each trial consisted of a 500 ms baseline, a $250 \mathrm{~ms} \mathrm{CS}$, a $500 \mathrm{~ms}$ trace period, a $150 \mathrm{~ms}$ US, and a $500 \mathrm{~ms}$ post-US period. Intertrial intervals varied between 30 and $60 \mathrm{~s}$, with an average of $45 \mathrm{~s}$. Rabbits were given $10 \mathrm{~d}$ of initial trace EBC (a total of 800 trials), which is sufficient training to acquire the learning task. After $30 \mathrm{~d}$ of rest, a surgical procedure, and $7 \mathrm{~d}$ of recovery, trace-conditioned rabbits received $5 \mathrm{~d}$ of retrieval sessions of trace EBC.

Pseudo-EBC. A total of three rabbits in the recording study underwent pseudo-EBC. These rabbits were set up exactly as the trace-conditioned rabbits. After $1 \mathrm{~d}$ of habituation, rabbits received daily sessions consisting of 160 unpaired CS alone or US alone trials, which varied randomly with an intertrial interval that ranged from 15 to $30 \mathrm{~s}$ with an average of $22 \mathrm{~s}$. These rabbits allowed us to measure the behavioral and neuronal response to unpredictable whisker stimulation and corneal air puffs in a nonlearning situation. Initial pseudo-EBC was performed for $10 \mathrm{~d}$. After $30 \mathrm{~d}$ of rest, a surgical procedure, and $7 \mathrm{~d}$ of recovery, these rabbits were given an additional $5 \mathrm{~d}$ of pseudo-EBC.

Delay EBC. All 14 rabbits in the lesion study were trained in and acquired delay EBC. Delay EBC is a control to ensure that cerebellar and brainstem nuclei are intact and that our training apparatus is properly set up. This control is useful in interpreting the results of a lesion study such as this one, when the lesions caused impairments in performance of trace-conditioned responses. The rabbits were set up exactly the same as trace-conditioned rabbits. Each daily session consisted of 80 trials; each trial consisted of a $900 \mathrm{~ms}$ CS and a $150 \mathrm{~ms}$ US that began 750 $\mathrm{ms}$ after CS onset and coterminated with the CS. The intertrial interval varied from 30 to $60 \mathrm{~s}$ with an average of $45 \mathrm{~s}$. The rabbits received $4 \mathrm{~d}$ of delay EBC (320 total trials) after retrieval sessions of trace EBC.

\section{Analyses}

Behavioral data analyses. All behavioral analyses were performed blind to the rabbits' surgical histories. For each trial, the baseline voltage was recorded for $250 \mathrm{~ms}$ before CS onset. Any movement of the nictitating membrane (third eyelid) during the CS-US interval that resulted in a change $>4$ SDs over pre-CS baseline was analyzed. Responses that returned to baseline before the onset of the US were considered poorly

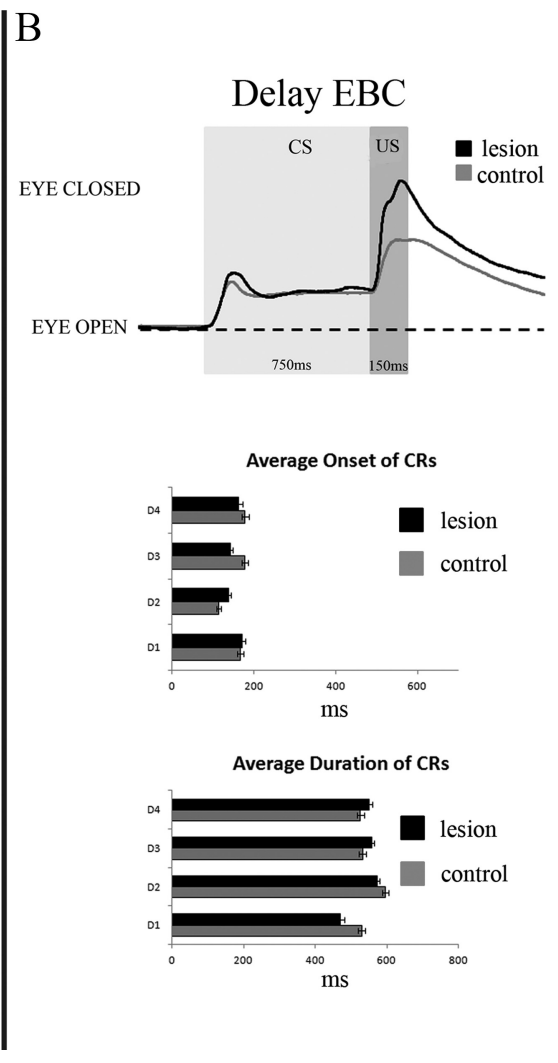

Figure 2. Characteristics of CRs performed by CN-lesioned and sham-lesioned rabbits. A, Top to bottom, Average CRs performed during $1 \mathrm{~d}$ of trace $E B C$ retrieval by a CN-lesioned rabbit (black) and a sham-lesioned rabbit (gray). The average $\mathrm{CR}$ of the $\mathrm{CN}$ 列 atween $\mathrm{CN}$ - and sham-lesioned rabits on any of the 5 d of trace $\mathrm{EBC}$ ritrieval (T1-T5), but the duration of av the average CRs of all seven $\mathrm{CN}$-lesioned and seven sham-lesioned rabbits across the final $3 \mathrm{~d}$ of delay $E B C$, with the only day of delay $E B C$ (D1).

timed or "non-adaptive" responses. Only responses that resulted in a 4 SD change during the $20 \mathrm{~ms}$ before US onset were considered appropriately timed "adaptive" CRs, because such CRs acted to reduce the impact of the air-puff US on the cornea (Perrett et al., 1993). The first day in which a rabbit performed eight adaptive CRs in 10 consecutive trials was considered the day of learning criterion in the recording study. To be considered a "learner" in the lesion study, rabbits must have achieved 2 consecutive days of eight adaptive CRs in 10 consecutive trials. Because there were fewer trials of delay EBC, learning criterion was reduced to $1 \mathrm{~d}$ of eight adaptive CRs in 10 consecutive trials for the delay paradigm. All rabbits, regardless of previous training or lesion group, achieved learning criterion in delay EBC within $4 \mathrm{~d}$ of training. A group $\times$ repeatedmeasures ANOVA was used to determine significant difference between the training groups.

Electrophysiological analyses. Single-neuron signals were amplified $(10,000 \times)$, filtered between $600 \mathrm{~Hz}$ and $6 \mathrm{kHz}$, and collected on a personal computer that sampled each channel at $32 \mathrm{kHz}$. Data were collected continuously using Cheetah Data Acquisition System (Neuralynx). Single action potentials were isolated using measurements of spike height and width using Spike Sort Cluster Cutting Tool from Neuralynx, generating two and three-dimensional scatter plots allowing for tracking the activity of a neuron throughout a single session. Cells recorded from each day were treated as individual neurons because the electrode was moved at the end of each day of trace EBC. Populations of neurons with similar characteristics were grouped together based on similar firing patterns, interspike interval, and firing rate. Medium spiny neurons (MSNs) were identified using previously established criteria to distinguish them from 
A

LEFT CAUDATE NUCLEUS

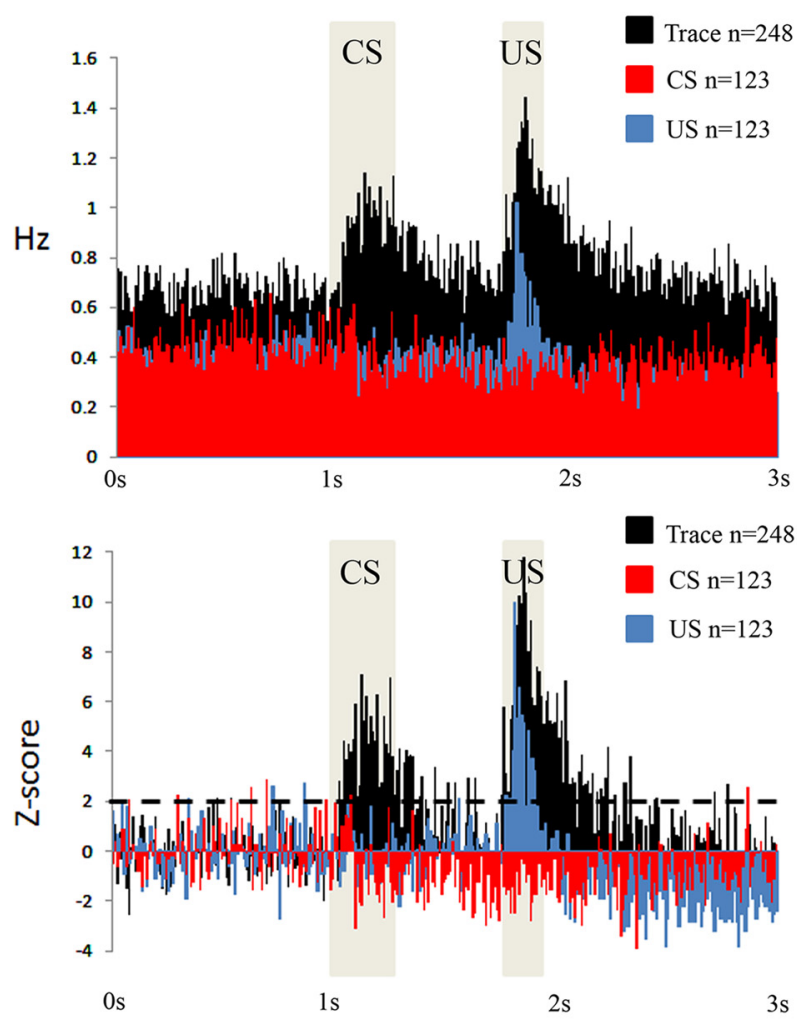

C

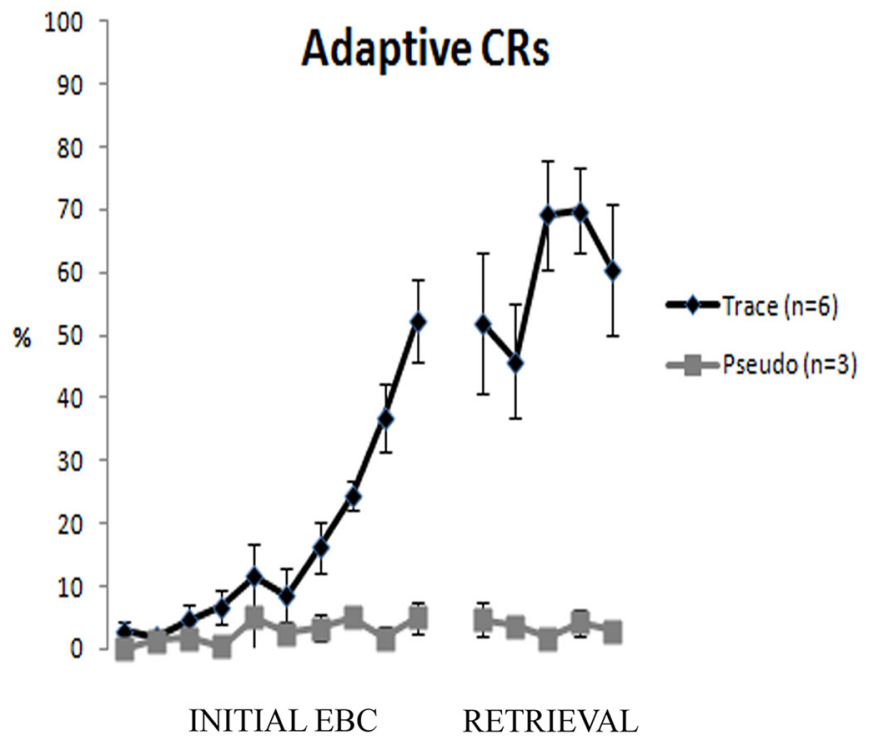

B

RIGHT CAUDATE NUCLEUS
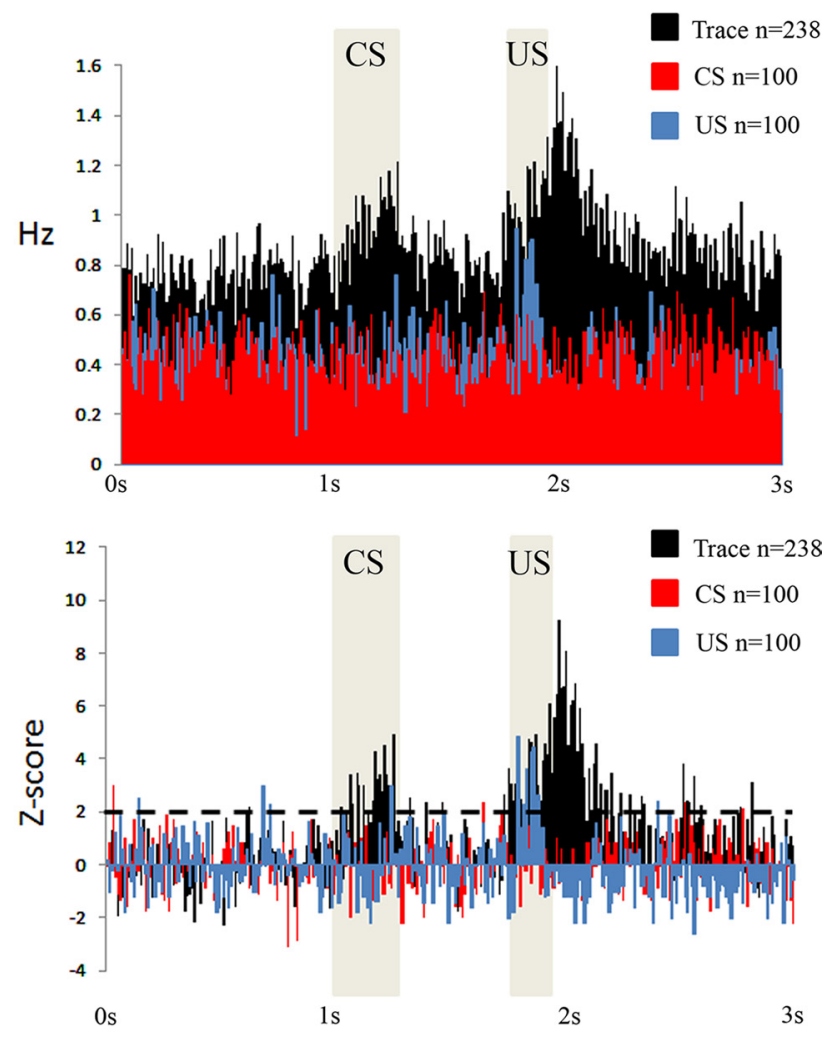

D

RATE INCREASING

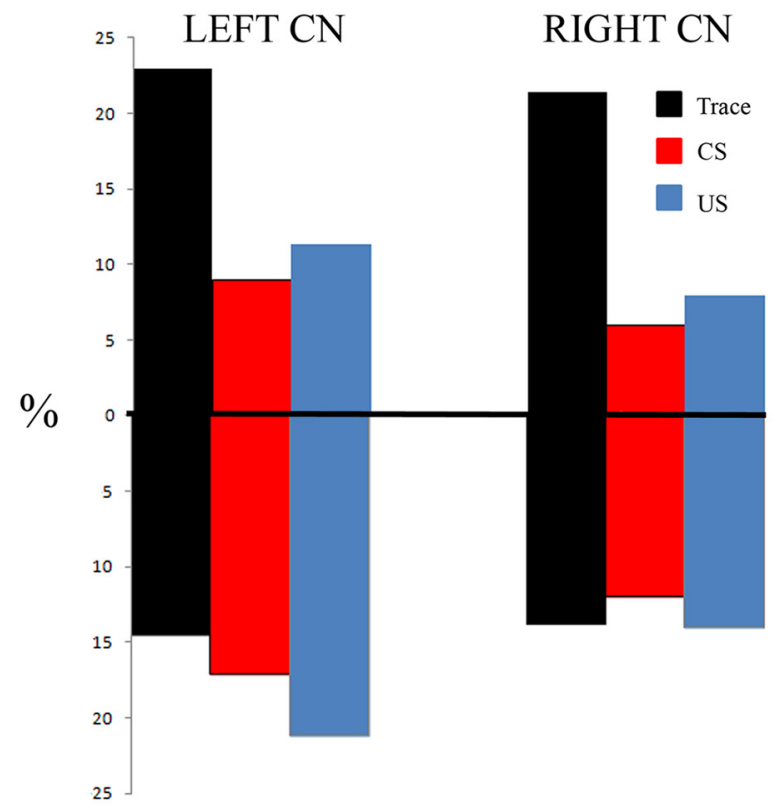

\section{RATE DECREASING}

Figure 3. MSNs are responsive to the CS and US during trace EBC retrieval. $A$, Combined activity of all presumptive MSN s recorded from the left CN (contralateral to the trained eye) during all 5 d of trace EBC shown in hertz and Z score. There is a strong response to the $C S$ that is present during trace $\mathrm{EBC}$ (black bars) that is not present during $\mathrm{CS}$ trials of pseudo-EBC (red bars). There is a response to the US during pseudo-EBC (blue bars). $B$, Combined activity of all presumptive MSNs recorded from the right $C \mathrm{~N}$ (ipsilateral to the trained eye) over $5 \mathrm{~d}$ of trace EBC shown in hertz and Zscore. There is a response to the CS during trace $\mathrm{EBC}$ (black bars) that is not present during (S trials of pseudo-EBC (red bars). There is a response to the US during pseudo- $\mathrm{EBC}$ (blue bars). The baseline firing rate is significantly higher during trace $\mathrm{EBC}$ than pseudo-EBC.C, AdaptiveCRs performed during initial trace and pseudo-EBC and EBC retrieval. Recordings took place during EBC retrieval. $D$, Percentage of total MSNs that significantly increased or decreased their firing rate during trace and pseudo-EBC in the left and right $\mathrm{CN}$. The percentage of MSNs that increase their firing rates is greater in both the left and right $\mathrm{CN}$ compared with pseudo-EBC. The percentages of rate-decreasing MSNs does not differ much with the exception of more rate-decreasing MSNs during US trials of pseudo-conditioning in the left CN. 
tonically active neurons (TANs; Flores and Disterhoft, 2009). Perievent time-stamped histograms and raster plots were built to analyze neuronal activity $1 \mathrm{~s}$ before and $2 \mathrm{~s}$ after CS onset using Neuroexplorer. For some analyses, the activity of neurons during trials in which a CR was separated from trials in which no CR was performed. To be included in this study, each neuron must have been histologically verified in the $\mathrm{CN}$ and passed a 2.5:1 signal/noise ratio. Mann-Whitney $U$ analysis was performed on each neuron to determine whether activity during the CS, trace, US, or post-US period significantly differed from the baseline period. $Z$ scores were calculated for neurons of interest using the following formula: $z=$ $(x-\mu) / \sigma$, where $x$ is the value each individual $10 \mathrm{~ms}$ bin, $\mu$ is the mean of all $10 \mathrm{~ms}$ bins of the baseline period, and $\sigma$ is the SD of all $10 \mathrm{~ms}$ bins of the baseline period.

Histology. In the recording study, marking lesions were made by passing direct current $(50 \mu \mathrm{A})$ for $5 \mathrm{~s}$ through each tetrode on the day before perfusion. Rabbits were anesthetized with a $1 \mathrm{ml}$ injection of Euthasol (390 mg pentobarbital sodium/50 mg phenytoin sodium per milliliter) into the left marginal ear vein and perfused through the heart with saline, followed by $10 \%$ Formalin in saline. The brains were extracted and immersed in $30 \%$ sucrose $/ 10 \%$ Formalin solution until they sank. The brains were blocked and embedded in Tissue-Tek OTC compound, sectioned at $75 \mu \mathrm{m}$ using a freezing stage microtome, mounted on gelatinsubbed slides, stained with cresyl violet and Prussian blue, and coverslipped. A light microscope was used to localize either CN lesion damage or electrode placement. $\mathrm{CN}$ lesion pictures were analyzed using $\mathrm{NIH}$ ImageJ. Pictures of the $\mathrm{CN}$ were taken at landmarks $\mathrm{AP}+4,+3,+2$, +1 , and $+0 \mathrm{~mm}$, based on a stereotaxic atlas (Girgis and Shih-Chang, 1981). These sites represented the head and rostral body of the CN. For the lesion study, the average size of the $\mathrm{CN}$ was measured and averaged for all control $\mathrm{CN}$ sections, and percentage of damage was determined for each lesioned rabbit by comparing with the average control $\mathrm{CN}$ size at each landmark. Postmortem analysis of tissue demonstrated that all seven lesioned rabbits received damage to the head and rostral body of the $\mathrm{CN}$ between 15 and $54 \%$.

\section{Results}

Rabbits with $\mathrm{CN}$ lesions display significantly fewer adaptive CRs than sham-lesioned rabbits during retrieval sessions of trace EBC

Fourteen rabbits underwent initial trace EBC. After $10 \mathrm{~d}$ of training, these rabbits were matched and separated into two groups to ensure that there was no significant difference in percentage of adaptive CRs (repeated-measures ANOVA, df $=1, F=0.495$, $p=0.4949$ ). This learning curve can be seen in Figure $1 A$ (labeled INITIAL TRACE EBC). After $30 \mathrm{~d}$ of rest, a lesion surgery, and $7 \mathrm{~d}$ of recovery, the rabbits were subjected to five retrieval sessions of trace EBC, which can been seen in Figure $1 A$ (labeled TRACE EBC RETRIEVAL). The CN-lesioned rabbits (Lesion) showed significantly fewer adaptive CRs than sham-lesioned rabbits (Control) (repeated-measures ANOVA, $\mathrm{df}=1, F=4.776, p=$ 0.0494 ). Examination of Figure $1 A$ shows that the control rabbits showed an increased number of adaptive CRs on the first day of remote testing and asymptoted at that level for the five postconsolidation testing days. In contrast, the lesioned rabbits showed the same number of adaptive CRs on the first testing day and did not increase their performance on successive remote testing days.

The lesioned and sham-lesioned rabbits showed a similar percentage of total responses (data not shown; repeated-measures ANOVA, $\mathrm{df}=1, F=1.413, p=0.2576)$. More than $30 \%$ of the responses performed by control and lesioned rabbits were nonadaptive on the final day of initial trace EBC (Fig. $1 B$ ). On the very first day of retrieval sessions, the percentage of non-adaptive responses performed by control rabbits dropped to $<15 \%$, whereas the percentage of non-adaptive responses in $\mathrm{CN}$-lesioned rabbits was unchanged from initial training. The $\mathrm{CN}$-lesioned rabbits

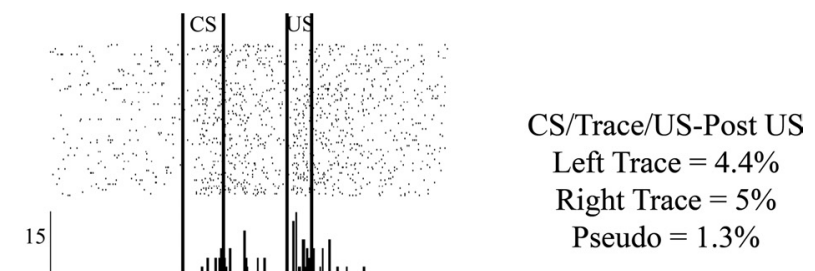

CS/US-Post US

Left Trace $=3.6 \%$

Right Trace $=2.9 \%$

Pseudo $=1.3 \%$

Trace/US-Post US

Left Trace $=2 \%$

Right Trace $=2.9 \%$

Pseudo $=1.8 \%$
US-Post US Only
Left Trace $=13.3 \%$
Right Trace $=5 \%$
Pseudo $=5.4 \%$

did not reduce the percentage of non-adaptive responses across the $5 \mathrm{~d}$ of retrieval sessions (Fig. $1 B$ ). The largest and smallest effective lesions resulted in similar deficits in adaptive CRs during retrieval of trace EBC (Figs. $1 C, D$ ). We analyzed our behavioral data to look for the possibility that there was rapid learning after the 1 month memory consolidation period. The sham-lesioned animals were at asymptote and showed no rapid learning at this point. Similarly, the CN-lesioned animals showed no shift in behavior with finer trial resolution.

In summary, the control rabbits increased their percentage of adaptive CRs and decreased their percentage of nonadaptive, short-latency responses in relation to their performance level at the end of initial training after 1 month of memory consolidation. The CN-lesioned rabbits showed performance levels that were unchanged from those at the end of initial training, in terms of both adaptive CRs and shortlatency, non-adaptive CRs. 


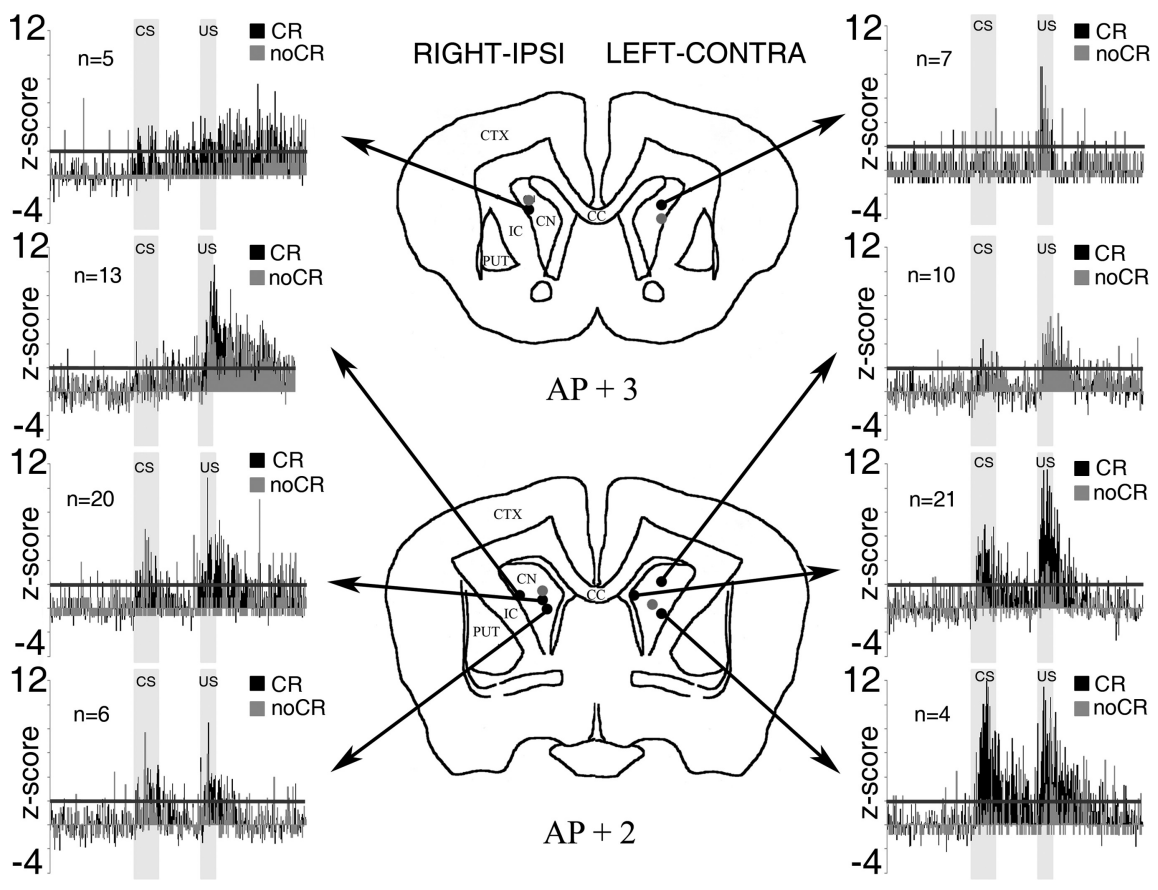

Figure 5. Activity from different regions of the left and right $C N$ during " $C R$ " and "noCR" trials of trace $E B C$ retrieval. Zscores from rate-increasing MSNs recorded from different regions of the $\mathrm{CN}$ during trace $\mathrm{EBC}$ demonstrate that medial and ventral regions of the $\mathrm{CN}$ are more responsive during the $\mathrm{CS}$ and trace period than dorsolateral regions. Furthermore, MSNs in medial and ventral regions of the $\mathrm{CN}$ were more responsive and showed more persistent activity during $\mathrm{CR}$ trials (black bars) than no-CR trials (gray bars). These increases were most obvious in the left $\mathrm{CN}$, contralateral to the conditioned eye. CC, Corpus callosum; CTX, cortex; IC, internal capsule; PUT, putamen.

The characteristics of all responses, both short-latency and adaptive CRs, performed by $\mathrm{CN}$ - and sham-lesioned rabbits were compared with each other. Figure $2 A$ shows the average of all behavioral trials performed on a typical single day of trace EBC retrieval by a control and a lesioned rabbit. The control rabbit performed an average CR that lasted throughout the trace period, whereas the average response performed by the lesioned rabbit returned to baseline before US onset, i.e., was non-adaptive. Averaging the responses performed by all rabbits showed that $\mathrm{CN}$ lesioned rabbits display responses that had similar onset times but shorter durations than sham-lesioned controls (Fig. 2A). This difference persisted through all $5 \mathrm{~d}$ of trace EBC retrieval.

\section{$\mathrm{CN}$-lesioned rabbits were unimpaired in delay EBC compared} with sham-lesioned rabbits

After $5 \mathrm{~d}$ of trace EBC, the rabbits were subjected to $4 \mathrm{~d}$ of delay EBC, which can be seen in Figure $1, A$ and $B$ (labeled DELAY $\mathrm{EBC}$ ). Interestingly, $\mathrm{CN}$-lesioned rabbits did not differ from sham-lesioned controls in adaptive CRs (repeated-measures ANOVA, $\mathrm{df}=1, F=1.019, p=0.3345)$. The same rabbit that had impaired timing during trace EBC retrieval performed delay EBC similarly to the sham-lesioned control (Fig. 2B). Analysis of all responses performed by lesioned and control rabbits during delay EBC demonstrated that both groups performed CRs with similar onset and duration times (Fig. 2B).

MSNs are responsive during retrieval sessions of trace EBC Figure $3 C$ shows the learning curves for six trace-conditioned rabbits and three pseudo-conditioned rabbits in both initial and retrieval sessions of trace and pseudo-EBC. At $30 \mathrm{~d}$ after this training, electrodes were implanted into the $\mathrm{CN}$, and retrieval sessions were performed after $7 \mathrm{~d}$ of recovery. Overall, 709 neu- rons met the criterion for MSNs over $5 \mathrm{~d}$ of recording: 248 from the left $\mathrm{CN}$ during trace $\mathrm{EBC}, 238$ from the right $\mathrm{CN}$ during trace $\mathrm{EBC}, 123$ from the left $\mathrm{CN}$ during pseudo-EBC, and 100 from the right $\mathrm{CN}$ during pseudo-EBC. Figure $3, A$ and $B$, shows the combined activity of MSNs in the left and right $\mathrm{CN}$ during $5 \mathrm{~d}$ of retention sessions of trace EBC and pseudo$\mathrm{EBC}$, respectively. The left $\mathrm{CN}$ was contralateral to the trained eye. There is a response to the CS that is present during trace EBC but absent during CS trials of pseudo-EBC in both the left and right $\mathrm{CN}$. MSNs in the $\mathrm{CN}$ do respond to the US during pseudo-EBC. Although the increase in baseline firing rate in traceconditioned MSNs in the right $\mathrm{CN}$ was significantly greater compared with pseudo-conditioned MSNs in the right $\mathrm{CN}$ (ANOVA, df $=1, F=6.741, p=$ $0.0098)$, the increase in baseline firing rate in the left $\mathrm{CN}$ only approached significance compared with pseudo-EBC (ANOVA, $\mathrm{df}=1, F=3.44, p=0.0645) . Z$ scores of all the neurons were calculated and averaged to see the magnitude of neuronal response relative to baseline activity. $Z$ scores in the left $\mathrm{CN}$ during trace EBC were higher than the right $\mathrm{CN}$.

Figure $3 D$ shows the percentages of neurons that significantly changed their firing rate during trace and pseudo-EBC in the left and right $\mathrm{CN}$. In both the right and left $\mathrm{CN}$, there was an increase in the percentage of MSNs that increased their rate of firing during trace EBC compared with pseudo-EBC. Rate-decreasing MSNs did not differ greatly during trace and pseudo-EBC, with the possible exception of a higher percentage of rate-decreasing MSNs in the left $\mathrm{CN}$ during pseudo-EBC.

Because trace $\mathrm{EBC}$ resulted in an increase in the percentage of rate-increasing neurons, these neurons were selected for additional analysis. Figure 4 shows the most prevalent response profiles of rate-increasing MSNs during trace EBC: CS/Trace/USPost US, CS/US-Post US, Trace/US-Post US, or US-Post US only. The percentage of total neurons belonging to each group was consistently higher in trace EBC compared with pseudo-EBC. This demonstrates that the increase in the percentage of rateincreasing MSNs in trace EBC was not specific to one particular response profile but was an increase in all different types of responsive MSNs.

\section{Regional specificity of responsive MSNs during trace EBC retrieval on $\mathrm{CR}$ and non-CR trials}

To compare our recording results with the behavioral results, we looked at the activity of MSNs that were recorded from different regions of the $\mathrm{CN}$ to see whether there were regional differences on trials when CRs occurred. Figure 5 shows $Z$ scores of rateincreasing MSNs from different regions of the right and left $\mathrm{CN}$ during trials in which a CR was performed and those in which the rabbit did not perform a CR. In both the right and left CN, MSNs in the medial and ventral CN were more responsive to the CS and US than MSNs in the dorsolateral CN. Importantly, significant sustained firing through the trace period can be seen in many 

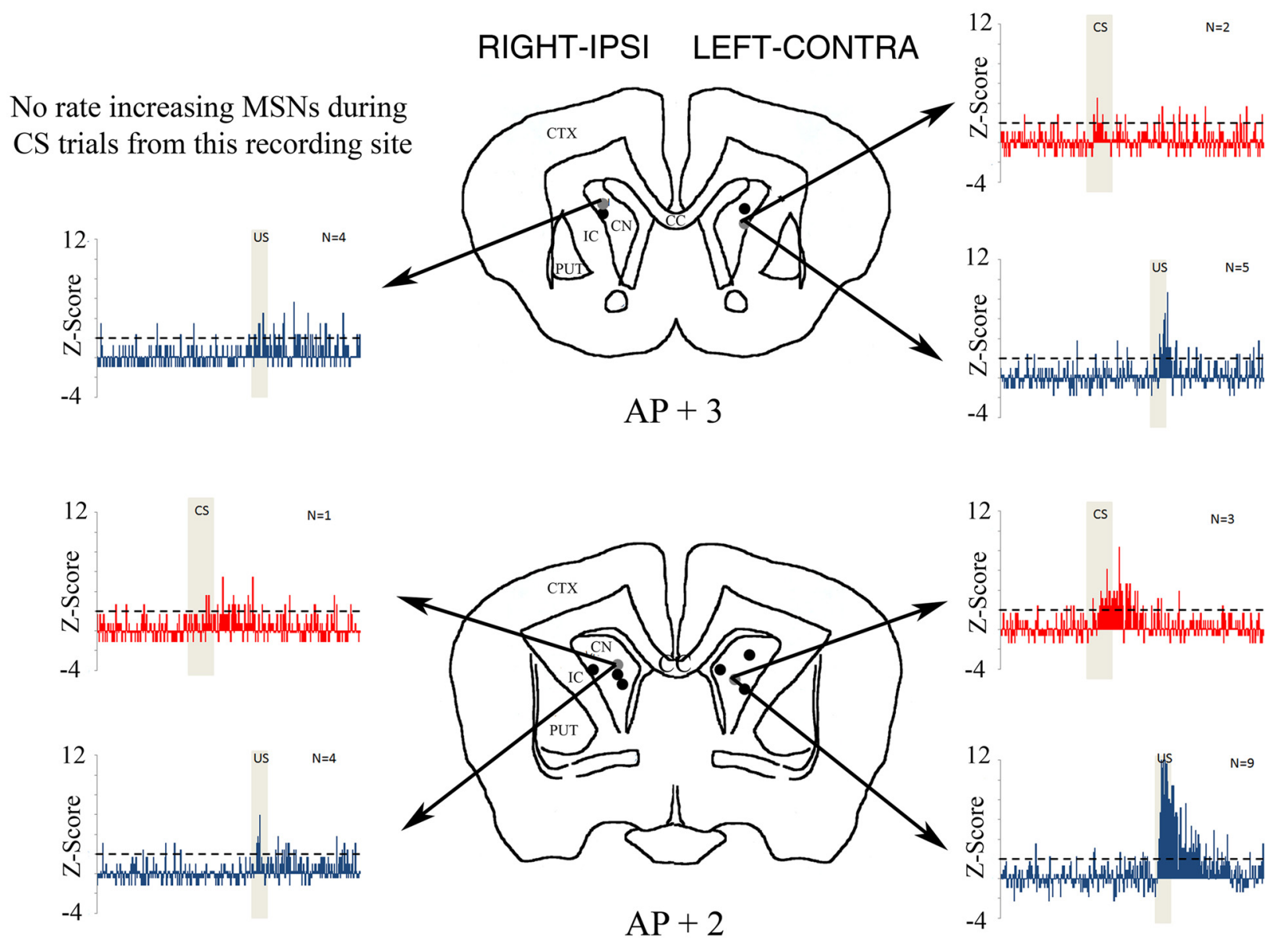

Figure 6. Activity from different regions of the left and right $\mathrm{CN}$ during pseudo-EBC.Z scores from the very few rate-increasing MSNs demonstrate very little activity during $C S$ trials of pseudo-EBC (red bars). There were more rate-increasing MSNs during US trials of pseudo-EBC (blue bars). CC, Corpus callosum; CTX, cortex; IC, internal capsule; PUT, putamen.

regions of the medial and ventral $\mathrm{CN}$ during "CR" trials but not during "noCR" trials. Figure 6 shows $Z$ scores from the few rateincreasing MSNs recorded during CS and US trials of pseudoEBC. The responses of these MSNs are smaller compared with the activity seen in similar regions during trace $\mathrm{EBC}$.

\section{Responsive TANs during trace $\mathrm{EBC}$ retrieval}

TANs represent a very small percentage of neurons in the CN, and only three well-isolated neurons matched criterion for TANs in these recordings. One of those TANs as well as neighboring MSNs are shown in Figure 7. The TAN "paused" its tonic firing in response to both the CS and US, whereas the MSN showed the opposite response profile. The waveform of the TAN was shorter than the MSN waveform, and the interspike interval of the TAN was shifted away from $0 \mathrm{~ms}$, indicative of a neuron with a long refractory period. The interspike interval of the MSN was very close to $0 \mathrm{~ms}$ because of its short refractory period and tendency to burst fire.

\section{Discussion}

The results from the current study indicate that the $\mathrm{CN}$ is still involved in trace EBC 1 month after the animals have acquired this learned association. Lesions of the $\mathrm{CN}$ made at that point blocked the ability of rabbits to refine their CRs compared with their level at the end of initial training, 1 month before the lesion.
Control rabbits increased their percentage of adaptive CRs and decreased their percentage of short-latency, non-adaptive responses during remote testing. Lesioned rabbits did not show these behavioral changes, but both types of responses stayed at their levels after initial training. The responses performed by $\mathrm{CN}$ lesioned rabbits were short latency and non-adaptive, they peaked too soon, and did not last throughout the trace period. This meant that the lesioned rabbits tended to receive the full impact of the air-puff US, because the eye was open at air-puff delivery. Sham-lesioned animals showed a higher percentage of adaptive CRs during the very first day of retrieval sessions of trace EBC compared with their own performance at the conclusion of the initial trace EBC, suggesting that memory consolidation took place during the rest period in the sham animals. This increase in adaptive CRs after memory consolidation was not seen in the CN-lesioned rabbits. The lesions in this study were made 1 month after initial training, a period chosen to allow memory consolidation processes to occur. It is possible that lesions made immediately after initial training would have caused a similar effect. Although in such a case it is doubtful that the shamlesioned animals would have been at asymptotic performance as quickly as observed here, the lesion-sham differences present after memory consolidation may not have been obvious without additional training. It would be necessary to do lesions immedi- 
A

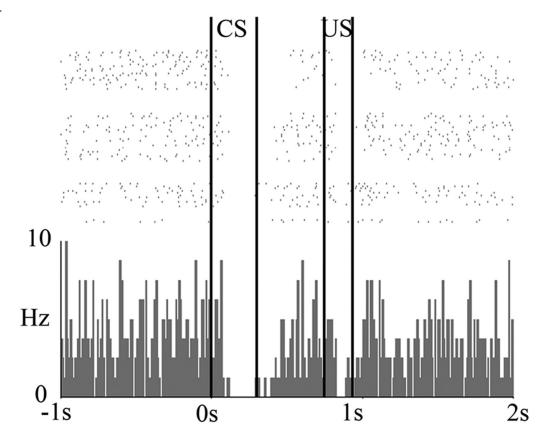

B

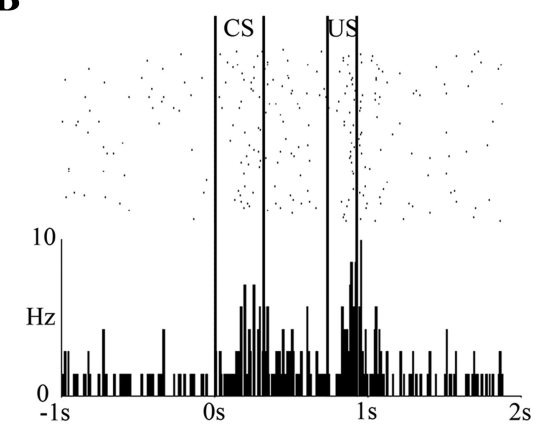

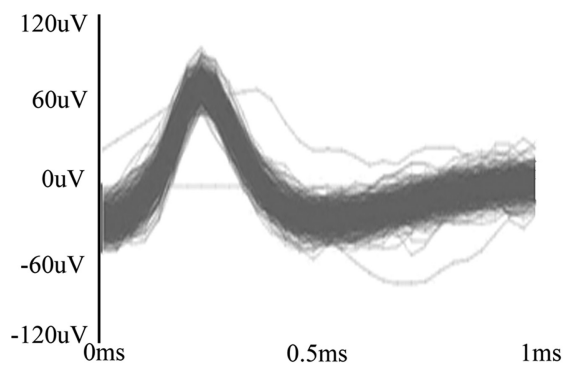
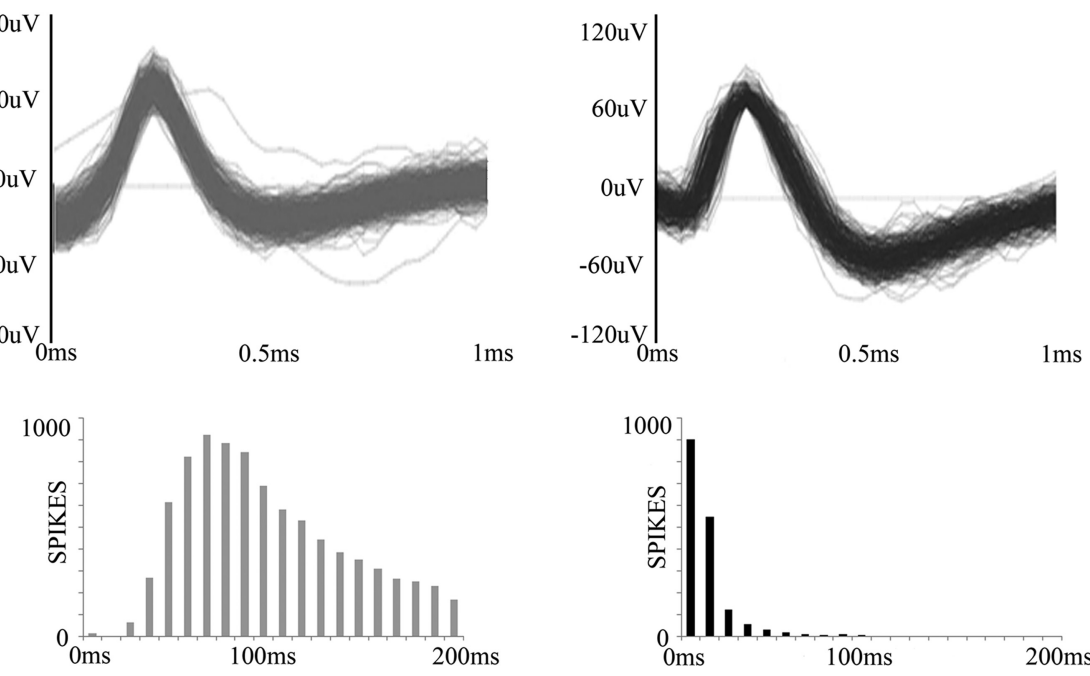

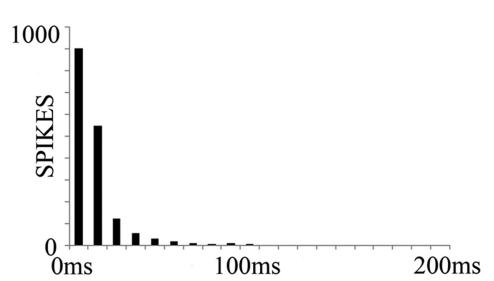

retrieval, control rabbits were greatly improved in their performance, suggesting that the CN-lesioned rabbits should have been performing at a greater level even without additional training, apparently as a result of the processes of memory consolidation. It is doubtful that lesion size was a factor in our data because the rabbits with the largest lesion (54\%) and smallest lesion (15\%) were similarly impaired during retrieval of trace $\mathrm{EBC}$.

$\mathrm{CN}$-lesioned rabbits were able to perform delay EBC similarly to shamlesioned rabbits with no deficit in either total or adaptive CRs. The CRs performed by $\mathrm{CN}$-lesioned rabbits improved in duration during delay EBC. This result was not surprising because the timing mechanism for performing CRs during delay EBC is in cerebellar networks (Kalmbach et al., 2010). This demonstrates that the memory consolidation impairment caused by $\mathrm{CN}$ lesions was specific to trace $\mathrm{EBC}$ and the lesion effect was not attributable to a generalized motor impairment.

The results of the recording study indicate that neurons in the $\mathrm{CN}$ were still responsive during retention trials of trace EBC. During pseudo-EBC, there was a response to the US, but not to the CS, in both the right and left $\mathrm{CN}$. This was an expected result that is similar to what was seen during initial pseudo-EBC in the $\mathrm{CN}$ (Flores and Disterhoft, 2009). Because trace eyeblink-conditioned rabbits had more rate-increasing MSNs, we chose to focus on these neurons for additional analysis. Many of these neurons not only increased their firing rate during the CS

Figure 7. $A C S / U S$ responsive TAN and a nearby responsive MSN recorded during trace EBC retrieval. $A$, Top to bottom, pt of a TAN during one session of trace EBC retrieval demonstrating a pause in tonic firing during the CS and US periods with waveform and interspike interval underneath. The interspike interval is a very effective method for identifying TANs, which characteristically have interspike intervals that are shifted away from $0 \mathrm{~ms}$. B, Top to bottom, Raster plot of an MSN recorded from the same tetrode bundle on the same day as the TAN shown in $A$. The MSN increases its firing rate in response to the CS and US in opposition to the pattern shown by the TAN. The recorded waveform and the interspike interval are below the raster plot. The interspike interval of the MSN peaks at $10 \mathrm{~ms}$, which is characteristic of burst firing MSNs.

ately after training to evaluate this possibility. In any case, the important point is that $\mathrm{CN}$-lesioned rabbits were markedly impaired in their ability to fine tune their CRs during remote testing.

The total number of behavioral responses and their onset times did not differ between $\mathrm{CN}$-lesioned and sham-lesioned rabbits, suggesting that the $\mathrm{CN}$ does not play a role in motor initiation of well-learned tasks, which is in agreement with other studies (Desmurget and Turner, 2008). The responses of the $\mathrm{CN}$-lesioned rabbits had shorter durations than sham-lesioned rabbits, suggesting that the $\mathrm{CN}$ scales the $\mathrm{CR}$ into an appropriate and adaptive response. This interpretation would be in agreement with a study that inactivated the internal globus pallidus in a well-learned motor task and found a problem with motor execution but not memory (Desmurget and Turner, 2010). If the CN were the only locus for the associative memory that was established previously during initial trace EBC, it would be expected that our lesion effect would have been larger. For example, lesions of the dorsomedial striatum (analogous to the $\mathrm{CN}$ in rabbits) prevented both acquisition and expression of operant conditioning in rats (Yin et al., 2005), but our lesion effect was less dramatic. The lesioned animals showed the same number of adaptive CRs and non-adaptive short-latency responses as during initial acquisition but were unable to further improve their performance after the lesion. However, on the first day of trace EBC and US periods but also increased their firing during the trace period as well. This trace period activity was most evident on trials when the rabbits demonstrated adaptive CRs. Persistent activity in the trace period has also been reported after learning trace $\mathrm{EBC}$ in the medial prefrontal cortex in rats (TakeharaNishiuchi and McNaughton, 2008) and rabbits (Siegel et al., 2012), regions that provide input into the CN (Weible et al., 2007). This activity could be important for the execution of appropriately timed CRs. If we assume that the rate-increasing MSNs are projecting preferentially via the direct pathway of the basal ganglia, then their activity would inhibit the firing of the output nuclei of the basal ganglia. Because the output nuclei of the basal ganglia are GABAergic TANs, this inhibition results in disinhibition of downstream targets, which could allow a CR initiated by the cortex to persist throughout the trace period instead of ending at CS offset. Recordings from the output nuclei of the direct and indirect output of the basal ganglia during trace EBC would need to be performed to determine whether this were the case.

The interaction between forebrain structures such as the CN and the cerebellum remains a puzzle, but a study of the role of cerebellar interpositus nucleus during trace conditioning with parallels to this one suggests that the $\mathrm{CN}$ must be interacting with the cerebellum in some important manner (Pakaprot et al., 
2009). Muscimol inactivation of the interpositus blocked expression of trace eyeblink-conditioned responses in rabbits either immediately after ( $1 \mathrm{~d}$ ) or remotely (32 d) after initial training. Interestingly, savings after muscimol activation were pointed out by the authors as suggesting the contribution of structures other than the cerebellum as playing a role in trace EBC learning and retention. Our findings suggest that the $\mathrm{CN}$ may well be one forebrain structure contributing importantly to this sensorimotor associative learning task when a persistent response is required during the trace interval.

MSNs in the CN have been demonstrated to be responsive during performance of well-learned tasks but that activity is usually diminished either in cell number or response magnitude compared with activity during acquisition (Barnes et al., 2005; Tang et al., 2009). One study of particular interest to our findings took recordings from the dorsolateral prefrontal cortex and the $\mathrm{CN}$ in monkeys well trained to perform a simple saccadic task (Jin et al., 2009). They found that individual MSNs with different activity patterns could form a population code of time together with neurons in the dorsolateral prefrontal cortex. A similar mechanism could explain the results of this study in which MSNs displayed different activity profiles, including persistent activity through the trace period, which might underlie the timing deficits seen in CN-lesioned rabbits during trace EBC retrieval, with the lesioned rabbits showing fewer adaptive responses and a larger number of short-latency non-adaptive responses. Although MSNs were the primary focus, a few TANs were recorded from and were still responsive during trace EBC retrieval. TANs have also been shown to remain responsive during a well-learned conditioning task even after a 1 month break in training in primates (Aosaki et al., 1994), which is in agreement with our findings here.

The $\mathrm{CN}$ appears to contribute to consolidation of trace $\mathrm{EBC}$ during remote retrieval. $\mathrm{CN}$ lesions before acquisition block the ability to learn (Flores and Disterhoft, 2009). Similarly, lesions before remote retrieval block the ability to improve learned performance relative to initial acquisition. Thus, $\mathrm{CN}$ appears to play a more significant role in remote retention than the hippocampus or SI cortex, in which posttraining lesions had no effect or partial and recoverable effects, respectively (Kim et al., 1995; Galvez et al., 2007). The role of the $\mathrm{CN}$ appears to be to maintain an initiated CR through the trace period.

\section{References}

Aosaki T, Tsubokawa H, Ishida A, Watanabe K, Graybiel AM, Kimura M (1994) Responses of tonically active neurons in the primate's striatum undergo systemic changes during behavioral sensorimotor conditioning. J Neurosci 14:3969-3984. Medline

Barnes TD, Kubota Y, Hu D, Jin DZ, Graybiel AM (2005) Activity of striatal neurons reflects dynamic encoding and recoding of procedural memories. Nature 437:1158-1161. CrossRef Medline

Clark GA, McCormick DA, Lavond DG, Thompson RF (1984) Effects of lesions of the cerebellar nuclei on conditioned behavioral and hippocampal neuronal responses. Brain Res 291:125-136. CrossRef Medline

Desmurget M, Turner RS (2008) Testing basal ganglia motor functions through reversible inactivations in the posterior internal globus pallidus. J Neurophysiol 99:1057-1076. CrossRef Medline
Desmurget M, Turner RS (2010) Motor sequences and the basal ganglia: kinematics, not habits. J Neurosci 30:7685-7690. CrossRef Medline

Flores LC, Disterhoft JF (2009) Caudate nucleus is critically involved in trace eyeblink conditioning. J Neurosci 29:14511-14520. CrossRef Medline

Galvez R, Weible AP, Disterhoft JF (2007) Cortical barrel lesions impair whisker-CS trace eyeblink conditioning. Learn Mem 14:94-100. CrossRef Medline

Girgis M, Shih-Chang W (1981) A new stereotaxic atlas of the rabbit brain. St. Louis, MO: Green.

Jin DZ, Fujii N, Graybiel AM (2009) Neural representation of time in cortico-basal ganglia circuits. Proc Natl Acad Sci U S A 106:1915619161. CrossRef Medline

Kalmbach BE, Davis T, Ohyama T, Riusech F, Nores WL, Mauk MD (2010) Cerebellar cortex contributions to the expression and timing of conditioned eyelid responses. J Neurophysiol 103:2039-2049. CrossRef Medline

Kim JJ, Clark RE, Thompson RF (1995) Hippocampectomy impairs the memory of recently, but not remotely, acquired trace eyeblink conditioned responses. Behav Neurosci 109:195-203. CrossRef Medline

Mauk MD, Thompson RF (1987) Retention of classically conditioned eyelid responses following acute decerebration. Brain Res 403:89-95. CrossRef Medline

McEchron MD, Disterhoft JF (1997) Sequence of single-unit changes in CA1 hippocampus during acquisition of trace eyeblink conditioned responses. J Neurophysiol 78:1030-1044. Medline

Moyer JR Jr, Deyo RA, Disterhoft JF (1990) Hippocampectomy disrupts trace eye-blink conditioning in rabbits. Behav Neurosci 104:243-252. CrossRef Medline

Moyer JR Jr, Thompson LT, Disterhoft JF (1996) Trace eyeblink conditioning increases CA1 excitability in a transient and learning-specific manner. J Neurosci 16:5536-5546. Medline

Packard MG, Knowlton BJ (2002) Learning and memory functions of the basal ganglia. Annu Rev Neurosci 25:563-593. CrossRef Medline

Pakaprot N, Kim S, Thompson RF (2009) The role of the cerebellar interpositus nucleus in short and long term memory for trace eyeblink conditioning. Behav Neurosci 123:54-61. CrossRef Medline

Perrett SP, Ruiz BP, Mauk MD (1993) Cerebellar cortex lesions disrupt learning-dependent timing of conditioned eyelid responses. J Neurosci 13:1708-1718. Medline

Siegel JJ, Kalmbach B, Chitwood RA, Mauk MD (2012) Persistant activity in a cortical-to subcortical circuit: bridging the temporal gap in trace eyelid conditioning. J Neurophysiol 107:50-64. CrossRef Medline

Solomon PR, Vander Schaaf ER, Thompson RF, Weisz DJ (1986) Hippocampus and trace conditioning of the rabbit's classically conditioned nictitating membrane responses. Behav Neurosci 100:729-744. CrossRef Medline

Takehara-Nishiuchi K, McNaughton BL (2008) Spontaneous changes of neocortical code for associative memory during consolidation. Science 322:960-963. CrossRef Medline

Tang CC, Root DH, Duke DC, Zhu Y, Teixeria K, Ma S, Barker DJ, West MO (2009) Decreased firing of striatal neurons related to licking during acquisition and overtraining of a licking task. J Neurosci 29: 13952-13961. CrossRef Medline

Weible AP, McEchron MD, Disterhoft JF (2000) Cortical involvement in acquisition and extinction of trace eyeblink conditioning. Behav Neurosci 114:1058-1067. CrossRef Medline

Weible AP, Weiss C, Disterhoft JF (2007) Connections of the caudate anterior cingulate cortex in rabbit neural circuitry participating in the acquisition of trace eyeblink conditioning. Neuroscience 145:288-302. CrossRef Medline

Yin HH, Ostlund SB, Knowlton BJ, Balleine BW (2005) The role of the dorsomedial striatum in instrumental conditioning. Eur J Neurosci 22: 513-523. CrossRef Medline 